\title{
Dynamics of two interacting Bose-Einstein condensates
}

\author{
A. Sinatra ${ }^{1}$, P.O. Fedichev ${ }^{2,3}$, Y. Castin ${ }^{1}$, J. Dalibard ${ }^{1}$, and G.V. Shlyapnikov ${ }^{1,2,3}$ \\ ${ }^{1}$ Laboratoire Kastler Brossel*, 24, Rue Lhomond, F-75231 Paris Cedex 05, France \\ ${ }^{2}$ FOM Institute for Atomic and Molecular Physics, Kruislaan 40\%, 1098 SJ Amsterdam, The Netherlands \\ ${ }^{3}$ Russian Research Center, Kurchatov Institute, Kurchatov Square, 123182 Moscow, Russia
}

(April 11, 2018)

\begin{abstract}
We analyze the dynamics of two trapped interacting Bose-Einstein condensates in the absence of thermal cloud and identify two regimes for the evolution: a regime of slow periodic oscillations and a regime of strong non-linear mixing leading to the damping of the relative motion of the condensates. We compare our predictions with an experiment recently performed at JILA.

03.75.Fi,05.30.Jp
\end{abstract}

The experimental evidence for Bose-Einstein condensation in trapped atomic gases [1] has attracted a lot of attention, as the presence of a macroscopically occupied quantum state makes the behavior of these gases drastically different from that of ordinary gas samples. Trapped Bose-Einstein condensates are well isolated from the environment and, at the same time, can be excited by deforming the trap or changing the interparticle interaction. The question of how the gas sample, being initially a pure condensate, subsequently reaches a new equilibrium state is directly related to the fundamental problem of the appearance of irreversibility in a quantum system with a large number of particles. Thus far the time dependent dynamics of trapped condensates has mainly been analyzed for a single condensate [2- 6 ] on the basis of the Gross-Pitaevskii equation for the condensate wavefunction. Remarkably, already in this mean field approach the stochastization in the condensate evolution has been found [3], and the damping of the condensate oscillations has been observed numerically [5]. However, the question of the formation of a thermal component, addressed in [3], has not been investigated.

In this paper we study the evolution of a richer system, a mixture of two interacting condensates ( $a$ and $b$ ), in the situation where initially the thermal cloud is absent. The properties of a static two-component trapped condensate, including the issue of spatial separation of the $a$ and $b$ components due to interparticle interaction [7.8, were investigated in [9]. The response of the system to small modulations of the trap frequency has also been studied numerically [10]. In our case the $a$ and $b$ condensates have initially the same density profile and are set into motion mostly by an abrupt displacement of the trap centers. The main goal of our work is to study the dynamics of spatial separation of the two condensates and analyze how the system can acquire statistical properties and reach a new equilibrium state. From a general point of view, we are facing the problem raised by Fermi, Pasta and Ulam [11]. They considered classical vibrations of a chain of coupled non-linear oscillators, to analyze the emergence of statistical properties in a system with a large number of degrees of freedom. As has been revealed later, the appearance of statistical prop- erties requires a sufficiently strong non-linearity leading to stochastization of motion [12], whereas for small nonlinearity the motion remains quasiperiodic (see e.g. [13]).

We consider a situation in which the two condensates $a$ and $b$ see harmonic trapping potentials of exactly the same shape, and the interparticle interactions characterized by the scattering lengths $a_{a a}, a_{a b}$ and $a_{b b}$ are close to each other. The control parameter, determining the possibilities of non-linear mixing and stochastization, is the relative displacement $z_{0}$ of the trap centers. We identify two regimes for the evolution. In the first one the relative motion of the condensates exhibits oscillations at a frequency much lower than the trap frequency $\omega$. In the other regime there is a strong non-linear mixing leading to the damping of the relative motion, and the system has a tendency to approach a new equilibrium state. We compare our predictions with the results of the JILA experiments [14.15] on a two-component condensate of ${ }^{87} \mathrm{Rb}$ atoms in the $F=1, m=-1$ and $F=2, m=1$ states. In these experiments the double condensate was prepared from a single condensate in the state $F=1, m=-1$ (a) by driving a two-photon transition which coherently transfers half of the atoms to the state $F=2, m=1(b)$.

We mostly perform our analysis in the mean field approach relying on the Gross-Pitaevskii equations for the wavefunctions $\phi_{a}$ and $\phi_{b}$ of the $a$ and $b$ condensates. This approach corresponds to the classical limit of the evolution of a quantum field, the subsequent corrections being proportional to a small parameter $\left(n a_{\varepsilon \varepsilon^{\prime}}^{3}\right)^{1 / 2}(n$ is the gas density) and, hence, manifesting themselves only on a rather large time scale. The two coupled Gross-Pitaevskii equations for $\phi_{a}$ and $\phi_{b}$ normalized to unity read

$$
i \hbar \partial_{t} \phi_{\varepsilon}=\left[-\frac{\hbar^{2} \Delta}{2 m}+U_{\varepsilon}-\mu+\sum_{\varepsilon^{\prime}=a, b} g_{\varepsilon \varepsilon^{\prime}} N_{\varepsilon^{\prime}}\left|\phi_{\varepsilon^{\prime}}\right|^{2}\right] \phi_{\varepsilon} .
$$

Here $g_{\varepsilon \varepsilon^{\prime}}=4 \pi \hbar^{2} a_{\varepsilon \varepsilon^{\prime}} / m$ are the coupling constants for elastic interaction between atoms in the states $\varepsilon$ and $\varepsilon^{\prime}, m$ is the atom mass, and $N_{\varepsilon}, U_{\varepsilon}$ are the number of atoms and trapping potential for the $\varepsilon$ condensate. As in the JILA experiment, we choose the initial condition $\phi_{a, b}(0)=\phi_{0}$, where the (real) wavefunction $\phi_{0}$ corresponds to the ground state of Eq.(1D) with all atoms in 
the $a$ state and no trap displacement. The chemical potential of this ground state is denoted as $\mu$.

We consider the $a$ and $b$ condensates in the ThomasFermi regime $(\hbar \omega \ll \mu)$ and assume the number of condensate atoms $N_{a}=N_{b}=N / 2$ 16]. The first set of our calculations is performed for the evolution of the condensates in a spherically symmetric trapping potential $U_{0}(r)=m \omega^{2} r^{2} / 2$ which at $t=0$ is displaced along the $z$ axis by a distance $z_{0} / 2$ for the $a$ atoms, and by $-z_{0} / 2$ for the $b$ atoms. We present the results for the time dependence of the mean separation between the condensates,

$$
u(t)=\int d^{3} r z\left(\left|\phi_{a}(\mathbf{r}, t)\right|^{2}-\left|\phi_{b}(\mathbf{r}, t)\right|^{2}\right) .
$$

For the curves in Fig. 1 the coupling constants are $g_{a a}=$ $g_{a b}=g_{b b}$, and for $z_{0}=0$ our initial state is an equilibrium state at $t \geq 0$. In this state the Thomas-Fermi radius of the condensate $R_{0}=\left(2 \mu / m \omega^{2}\right)^{1 / 2}$ serves as unit of length, and the shape of $\phi_{0}$ is determined by $\mu / \hbar \omega$. Hence, for $z_{0} \neq 0$ the dependence of the quantity $u / R_{0}$ on $\omega t$ is governed by the parameters $\mu / \hbar \omega$ and $z_{0} / R_{0}$.
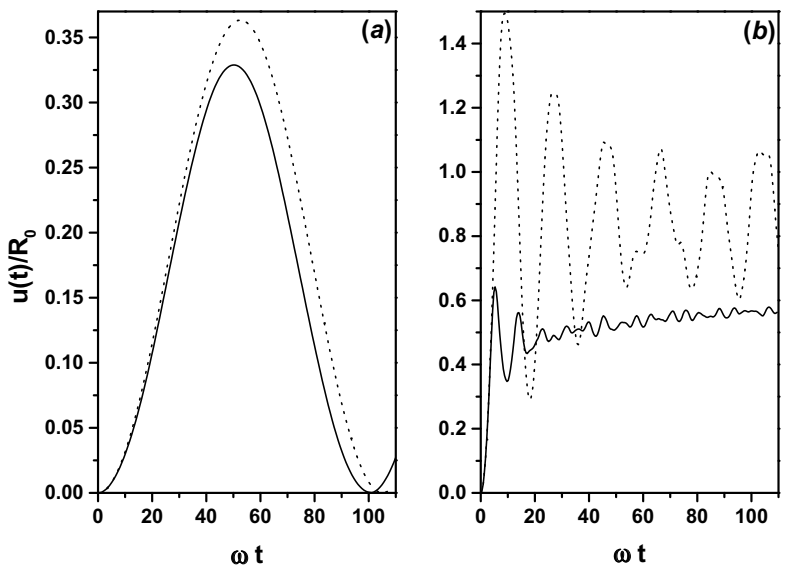

FIG. 1. Mean separation between the condensates versus time in isotropic traps for $g_{a a}=g_{a b}=g_{b b}$ and $\mu / \hbar \omega=29.2$. Relative displacement: $z_{0}=6.66 \times 10^{-4} R_{0}$ (a), and $z_{0}=7.16 \times 10^{-2} R_{0}(\mathrm{~b})$. Solid curves: numerical integration of Eq.(1). Dotted curves: analytical prediction for (a) (see text), and the linear model relying on Eq.(6) for (b).

Our results reveal two key features of the evolution dynamics. The first one, for a tiny displacement $z_{0}$, is a periodic motion with slow frequencies which turn out to be sensitive to small variations in the values of the coupling constants. The other feature, for much larger $z_{0}$, but still $z_{0} \ll R_{0}$, is a strong damping in the relative motion of the two condensates, as observed at JILA [14].

In order to understand the physics behind the evolution pattern, we first perform a linear analysis of Eq.(1D). For the case where $g_{a a}=g_{a b}=g_{b b}=g$, and the displacement $z_{0}$ is sufficiently small, we linearize Eq.(11) with respect to small quantities $\delta \phi_{a, b}=\left(\phi_{a, b}-\phi_{0}\right)$ and $z_{0}$. Then, for the quantity $\delta \phi_{-}=\delta \phi_{a}-\delta \phi_{b}$, describing the relative motion of the condensates, we obtain the equation

$$
i \hbar \partial_{t} \delta \phi_{-}=\left[-\frac{\hbar^{2} \Delta}{2 m}+U_{0}-\mu+N g \phi_{0}^{2}\right] \delta \phi_{-}+S_{-},
$$

with the source term $S_{-}=m \omega^{2} z_{0} z \phi_{0}$. For the quantity $\delta \phi_{+}=\delta \phi_{a}+\delta \phi_{b}$ we find an equation decoupled from $\delta \phi_{-}$ and without source terms. Hence, the initial condition $\delta \phi_{+}(\mathbf{r}, 0)=0$ allows us to put $\delta \phi_{+}(\mathbf{r}, t)=0$ for $t \geq 0$.

For $S_{-}=0$ Eq.(3) is the equation for the wavefunction of a particle moving in the potential $V=U_{0}-\mu+N g \phi_{0}^{2}$. Stationary solutions of this equation provide us with the eigenmodes of oscillations of the condensates with respect to each other. In the Thomas-Fermi limit the potential $V$, originating from the kinetic energy of the condensate, is a smooth function of $r$ inside the condensate spatial region $r<R_{0}: V=\hbar^{2}\left(\Delta \phi_{0}\right) / 2 m \phi_{0} \ll \hbar \omega$. For $r>R_{0}$ this potential is close to $U_{0}-\mu$ and is much steeper. Replacing $V$ by an infinite square well of radius $R_{0}$ we obtain the energy spectrum of eigenmodes with large quantum numbers $n: E_{n, l}=(\pi \hbar \omega)^{2}(2 n+l)^{2} / 16 \mu$, where $l$ is the orbital angular momentum. This explains the appearance of oscillations at a frequency much smaller than $\omega$ in our numerical calculations (see Fig.1a), since the energy scale in the spectrum is $(\hbar \omega)^{2} / \mu \ll \hbar \omega$. For the latter reason we call these eigenmodes soft modes. Note that the soft modes for the relative motion of the two condensates also exist in the spatially homogeneous case where they have a free-particle spectrum [7].

As in our linear approach we have $\delta \phi_{+}(\mathbf{r}, t)=0$, Eq.(2) for the mean separation between the condensates reduces to $u(t)=2 \int d^{3} r z \phi_{0} \operatorname{Re}\left\{\delta \phi_{-}\right\}$, and the contribution to $u(t)$ comes from the components of $\delta \phi_{-}$with $l=1, m_{l}=$ 0 . Solving Eq.(3) with the initial condition $\delta \phi_{-}(\mathbf{r}, 0)=0$, we obtain $u(t)$ as a superposition of components, each of them oscillating at an eigenfrequency of a soft mode:

$$
u(t)=z_{0} \sum_{n \geq 1} \frac{2 m \omega^{2}}{E_{n 1}}\left|\int d^{3} r \varphi_{n 1} z \phi_{0}\right|^{2}\left[1-\cos \left(\frac{E_{n 1} t}{\hbar}\right)\right],
$$

where $\varphi_{n 1}$ is the wavefunction of the soft mode with $l=1, m_{l}=0$ and main quantum number $n$. Damping of oscillations of $u(t)$ could, in principle, originate from the interference between the components with different $n$ in Eq.(四). However, the source $S_{-}$basically populates only the lowest soft mode, irrespective of the value of $z_{0}$ : the amplitude of oscillations at the lowest eigenfrequency in Eq.(1) (the term with $n=1$ ) greatly exceeds the sum of the amplitudes of other terms. Hence, these oscillations remain undamped. For the same reason their frequency and amplitude can be found with $\varphi_{n 1}$ replaced by the function $z \phi_{0}$ normalized to unity. Using the ThomasFermi approximation for the condensate wavefunction 17]: $\phi_{0}^{2}(r)=15\left(1-r^{2} / R_{0}^{2}\right) / 8 \pi R_{0}^{3}$ for $r<R_{0}$, and $\phi_{0}=0$ for $r>R_{0}$, we obtain $E_{11} \equiv \hbar \Omega=(7 / 4)(\hbar \omega)^{2} / \mu$ which is very close to $E_{11}=1.62(\hbar \omega)^{2} / \mu$ calculated numerically. Then, retaining only the leading term $(n=1)$ in Eq.(何), we find $u(t) \approx z_{0}(4 \mu / 7 \hbar \omega)^{2}[1-\cos (\Omega t)]$ shown in dotted 
line in Fig.1a. As one can see, the condition of the linear regime $u \ll R_{0}$ requires a very small displacement

$$
z_{0} \ll(\hbar \omega / \mu)^{2} R_{0},
$$

and already a moderate $z_{0}$ as in Fig.1b is sufficient to drive the system out of the linear regime.

We have performed a similar linear analysis for the case where $g_{a a} \neq g_{a b} \neq g_{b b}$, but the relative difference between the coupling constants is small. Also in this case the source $S_{-}$mostly generates oscillations of the condensates relative to each other at a single frequency $\Omega^{\prime} \ll \omega$. For a relative difference between the coupling constants much smaller than $(\hbar \omega / \mu)^{2}$, the frequency $\Omega^{\prime}$ coincides with the soft-mode frequency $\Omega$ found above. Otherwise the sign of $g_{-}=g_{a a}+g_{b b}-2 g_{a b}$ becomes important. In particular, for positive $g_{-} \gg\left|g_{a a}-g_{b b}\right|$ already a moderate difference between the coupling constants strongly increases the frequency $\Omega^{\prime}$ compared to $\Omega$. In this case we obtain undamped oscillations at $\Omega^{\prime} \approx\left(g_{-} / g_{a a}\right)^{1 / 2} \omega$. For $g_{-}<0$, already in the $z_{0}=0$ case, a breathing mode in which the two condensates oscillate out of phase becomes unstable, and the system evolves far from the initial state. Note that for a small difference between the coupling constants the condition $g_{-}<0$ is equivalent to the criterion of spatial separation of the condensates in the homogeneous case, $g_{a a} g_{b b}<g_{a b}^{2}$ [7]

We now turn to the large $z_{0}$ regime (Fig.1b) where we find a strong damping of the oscillations of the mean separation between the condensates, $u(t)$. In order to prove the key role of non-linearity in this regime, we first attempt a linear model assuming that the densities $\left|\phi_{\varepsilon^{\prime}}\right|^{2}$ inside the square brackets of Eq.(1) are not evolving:

$$
\sum_{\varepsilon^{\prime}} N_{\varepsilon^{\prime}} g_{\varepsilon \varepsilon^{\prime}}\left|\phi_{\varepsilon^{\prime}}\right|^{2} \rightarrow N g\left|\phi_{0}\right|^{2}
$$

In contrast to the analysis which led to Eq.(何), the displacement $z_{0}$ is now explicitly included in the Hamiltonian through the terms $\pm m \omega^{2} z z_{0} / 2$ in $U_{a, b}$, and the number of populated oscillation modes depends on $z_{0}$. However, for the parameters in Fig.1b we find that only a few modes are populated, and the interference between them can not account for the damping found numerically (dotted versus solid curve in Fig. 1b).

We argue that the damping in our calculations mostly originates from non-linearity of the system, which increases the number and amplitude of populated oscillation modes and provides an interaction between them. As a result, the evolution of the condensate wavefunctions $\phi_{a}$ and $\phi_{b}$ becomes chaotic. This can be seen from Fig.2 where we compare the spectral density $R_{n}(\nu)=$ $\left|T^{-1} \int_{0}^{T} d t n(\mathbf{0}, t) \exp (i \nu t)\right|^{2}$ of the density at the origin $n(\mathbf{0}, t)$ with an identically defined spectral density $R_{u}(\nu)$ of $u(t)$ for the parameters in Fig.1b and $T=110 / \omega$. The function $R_{n}(\nu)$ has a smooth envelope at large $\nu$, with peaks corresponding to the islands of regular motion. On the contrary, $R_{u}(\nu)$ exhibits pronounced peaks at $\nu$ of order $\omega$, without any smooth background. This picture provides a clear signature of stochastization in the system 13] and prompts us to represent each of the condensate wavefunctions in Eq.(11) as a superposition of two constituents: (i) a slowly oscillating regular part conserving the phase coherence properties; (ii) a composition of high-energy excitations characterized by stochastic motion. Only the slow constituent contributes to such macroscopic quantities as $u(t)$, since the contribution of the fast stochastic part is averaged out.

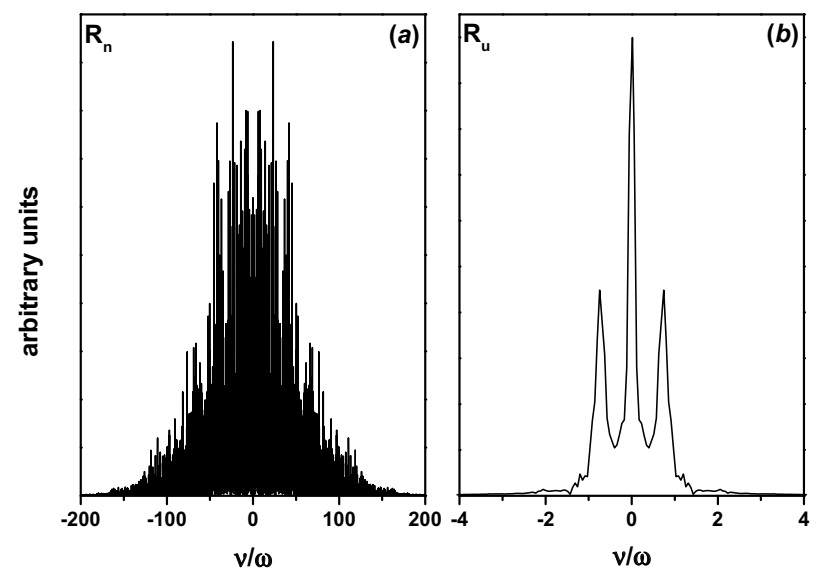

FIG. 2. Spectral densities $R_{n}(\nu)$ (a) and $R_{u}(\nu)$ (b) for the parameters in Fig. $1 \mathrm{~b}$ and $T=110 / \omega$ (see text).

Our analysis is consistent with the general statement that for a large population of various oscillation modes the non-linear interaction between them leads to stochastization in the motion of excitations with sufficiently high energy 13. This allows us to employ the mechanism of stochastic heating [13] for explaining the damping of oscillations of $u(t)$ : The mean field interaction between the fast stochastic and the slowly oscillating parts leads to energy transfer from the slow to the fast part.

The evolution of the occupation numbers of the modes of the fast stochastic part is governed by kinetic equations [13] and eventually slows down. The rate of energy and particle exchange between the two constituents then reduces. After a sufficiently long time only small linear oscillations of the condensates survive, mostly at the lowest eigenfrequency and the gas sample as a whole could be thought as being close to a steady state. However the damping of the remaining oscillations and the ultimate evolution of the fast stochastic part towards the thermal equilibrium require an analysis beyond the mean field approach. For the parameters in Fig.1b, using the semiclassical Bogolyubov approach 18 and relying on the conservation of energy and number of particles, we find an equilibrium temperature $T_{\mathrm{eq}} \approx 0.6 \mu$ and a condensed fraction $\gamma_{a, b} \approx 0.9$, for $N=5 \times 10^{5}$.

The last set of our calculations relates to the recent JILA experiment [14] where the evolution of a twocomponent ${ }^{87} \mathrm{Rb}$ condensate has been investigated. In 
the conditions of this experiment we solved numerically Eq.(1) by taking $a_{a b}=55 \AA$ and the ratio $g_{a a}: g_{a b}: g_{b b}=$ $1.03: 1: 0.97$. We also explicitly included in these equations the $22 \mathrm{~ms}$ expansion of the clouds after switching off the trapping potential. The results of our calculations are presented in Fig.3. As in Fig.1b, we find a strong damping of the oscillations of the mean separation between the condensates, $u(t)$. Our numerical results are in fair agreement with the experimental data, although the damping in the experiment is somewhat larger. We extended the calculations to twice the maximum experimental time and found small oscillations which remain undamped on this time scale.

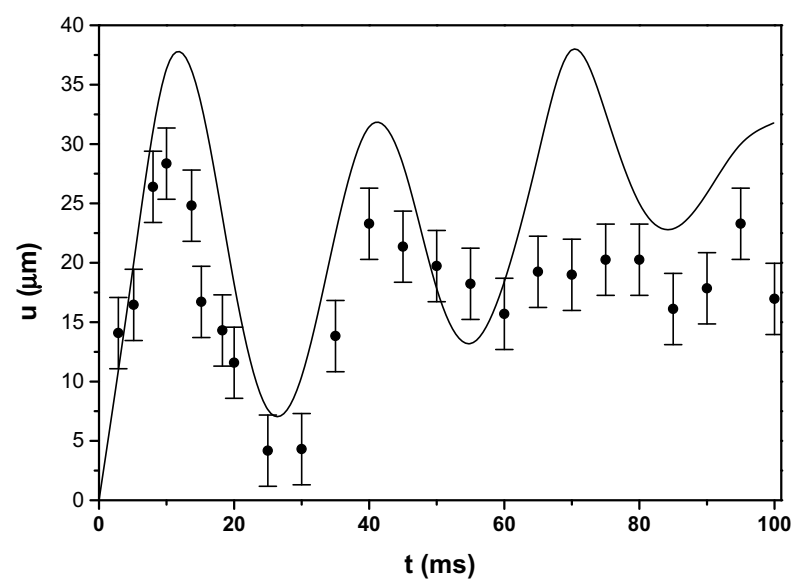

FIG. 3. Mean separation between the condensates in the JILA experiment versus evolution time in the traps, after a 22 ms free expansion. Dots with error bars: JILA experiment. Solid curve: our numerical calculation.

Our data for the JILA experiment 14 can be analyzed along the same lines as the results in Fig.1b, with a damping originating from stochastization in the evolution of the condensate wavefunctions. The equilibrium temperature is close to $\mu$, corresponding to condensed fractions $\gamma_{a} \approx \gamma_{b} \approx 0.9$. The large value of the condensed fraction explains why phase coherence between the $a$ and $b$ components could be observed even after the damping of the motion of $u(t)$ [15]. The damping time of the small remaining oscillations, estimated along the lines of [19], will be of order 1 second.

We believe that the stochastic regime identified from our calculations is promising for investigating the loss of phase coherence and the formation of a new thermal component in initially purely Bose-condensed gas samples. An interesting possibility concerns the observation of a continuous change in the phase coherence between the $a$ and $b$ components with increasing the trap displacement and, hence, decreasing the final Bose-condensed fraction.

This work was partially funded by EC (TMR network ERB FMRX-CT96-0002). by the Stichting voor Fundamenteel Onderzoek der Materie (FOM), by the Russian Foundation for Basic Studies, by INTAS, and by NSF under Grant No. PHY94-07194.
${ }^{*}$ L. K. B. is an unité de recherche de l'Ecole Normale Supérieure et de l'Université Pierre et Marie Curie, associée au CNRS.

[1] M. H. Anderson, J. R. Ensher, M. R. Matthews, C. E. Wieman, and E. A. Cornell, Science 269, 198 (1995); K. Davis, M.-O. Mewes, M. R. Andrews, N. J. van Druten, D. S. Durfee, D. M. Kurn and W. Ketterle, Phys. Rev. Lett. 75, 3969 (1995); C. C. Bradley, C. A. Sackett, and R. G. Hulet, Phys. Rev. Lett. 78, 985 (1997).

[2] P. A. Ruprecht, M. J. Holland, K. Burnett, and M. Edwards, Phys. Rev. A 51, 4704 (1995).

[3] Y. Kagan, E. L. Surkov, and G. Shlyapnikov, Phys. Rev. A. 54, R1753 (1996); 55, R18 (1997).

[4] Y. Castin and R. Dum, Phys. Rev. Lett. 77, 5315 (1996).

[5] A. Smerzi and S. Fantoni, Phys. Rev. Lett. 78, 3589 (1997).

[6] M. J. Holland, D. S. Jin, M. L. Chiofalo, and J. Cooper, Phys. Rev. Lett. 78, 3801 (1997).

[7] W.B. Colson and A. Fetter, J. Low Temp. Phys. 33, 231 (1978).

[8] E. Goldstein and P. Meystre, Phys. Rev. A 55, p.2935 (1997)

[9] T.-L. Ho and V. B. Shenoy, Phys. Rev. Lett. 77, 3276 (1996); B.D. Esry, C.H. Green, J.P. Burke, and J.L. Bohn, Phys. Rev. Lett. 78, 3594 (1997); C.K. Law, H. $\mathrm{Pu}$, N.P. Bigelow, and J.H. Eberly, Phys. Rev. Lett. 79, 3105 (1997); H. Pu and N.P. Bigelow, Phys. Rev. Lett. 80, 1130 (1998).

[10] H. Pu and N. P. Bigelow, Phys. Rev. Lett. 80, 1134 (1998).

[11] E. Fermi, J. Pasta and S. Ulam, in Collected papers of Enrico Fermi, Vol. II p.978, Accademia nazionale dei Lincei and University of Chicago, Roma (1965).

[12] F. M. Izrailev and B.V. Chirikov, Soviet Physics-Doklady 11, 30 (1966).

[13] R. Z. Sagdeev, D. A. Usikov, and G. M. Zaslavsky, Nonlinear Physics: From the Pendulum to Turbulence and Chaos, in Contemporary Concepts in Physics (Harwood Acad. Pub., Chur-New York, 1988).

[14] D. S. Hall, M. R. Matthews, J. R. Ensher, C. E. Wieman, E.A. Cornell, Phys. Rev. Lett. 81, 1539 (1998).

[15] D. S. Hall, M. R. Matthews, C. E. Wieman, and E. A. Cornell, Phys. Rev. Lett. 81, 1543 (1998).

[16] We solve Eq.(1) numerically, using cylindrical symmetry, on a finite grid with a splitting technique. Numerical accuracy is tested by changing the grid size and checking energy conservation $\left(\delta E / E \leq 10^{-5}\right.$ for $\left.\omega t=100\right)$.

[17] V. V. Goldman, I. F. Silvera, and A. J. Leggett, Phys. Rev. B 24, 2870 (1981); D. A. Huse and E. D. Siggia, J. Low Temp. Phys. 46, 137 (1982).

[18] F. Dalfovo, S. Giorgini, L. Pitaevskii, S. Stringari, Rev. Mod. Phys. 71, April 1999.

[19] P. O. Fedichev, G. V. Shlyapnikov, and J. T. M. Walraven, Phys. Rev. Lett. 80, 2269 (1998), and refs. therein. 